\title{
AN INEQUALITY FOR A QUADRATIC FUNCTIONAL
}

\author{
K.C. Le \\ Lehrstuhl für Allgemeine Mechanik, \\ Ruhr-Universität Bochum, 44780 Bochum, Germany
}

\begin{abstract}
An inequality is proved for a quadratic functional with the logarithmic kernel. The best constant of this inequality and the corresponding function for which the equality holds are found precisely.
\end{abstract}

The aim of this short communication is to provide one inequality for the following quadratic functional

$$
-\int_{a}^{b} \int_{a}^{b} \ln |x-y| \varphi^{\prime}(x) \varphi^{\prime}(y) d x d y,
$$

where $\varphi(x)$ are functions defined on the finite interval $(a, b)$ such that

$$
\varphi(x)=\phi(x) w(x), \quad w(x)=\sqrt{(b-x)(x-a)},
$$

with $\phi(x) \in W^{1,2}(a, b)$. Let $\tilde{W}(a, b)$ be the space of all such functions. The norm in this function space is defined as the weighted norm

$$
\|\varphi\|_{\tilde{W}}=\|\varphi / w\|_{W^{1,2}}=\|\phi\|_{W^{1,2}} .
$$

The quadratic functional (1) appears in connection with various problems of physics and mechanics, for instance, the crack problems $[1,2,3,4,5,6]$, the dislocation pile-up problems $[7,8,9]$, and the Peierls-Nabarro and Benjamin-Ono equations (see $[10,11]$ and the references therein). Because of the singularity of the logarithmic kernel, the double integral in (1) should be defined as

$$
-\int_{a}^{b} \int_{a}^{b} \ln |x-y| \varphi^{\prime}(x) \varphi^{\prime}(y) d x d y=-\lim _{\varepsilon \rightarrow 0} \int_{S_{\varepsilon}} \ln |x-y| \varphi^{\prime}(x) \varphi^{\prime}(y) d x d y,
$$

where $S_{\varepsilon}$ is the square $(a, b) \times(a, b)$ in the $(x, y)$-plane with the diagonal band of height $2 \varepsilon$ being removed

$$
S_{\varepsilon}=\{(x, y)|a<x<b, a<y<b,| x-y \mid>\varepsilon\} .
$$

Note that, by the partial integration with respect to $x$, one can present (2) in the form

$$
-\int_{a}^{b} \int_{a}^{b} \ln |x-y| \varphi^{\prime}(x) \varphi^{\prime}(y) d x d y=-\int_{a}^{b} f_{a}^{b} \frac{\varphi^{\prime}(y)}{y-x} d y \varphi(x) d x
$$


where $f$ denotes Cauchy's principal value of the integral. Note also the close connection of (3) with the capacity of the logarithmic potential induced by a 2-D continuous charge distribution on the interval $(a, b)$.

It turns out that the following inequality holds true for this functional: there exists a positive constant $\alpha>0$ such that for all $\varphi \in \tilde{W}(a, b)$

$$
-\int_{a}^{b} \int_{a}^{b} \ln |x-y| \varphi^{\prime}(x) \varphi^{\prime}(y) d x d y \geq \alpha \int_{a}^{b} \varphi^{2}(x) d x .
$$

By changing the variable $x \mapsto(2 x-a-b) /(b-a)$ if needed one can achieve that $a=-1$ and $b=1$. Therefore, to prove (4) it is sufficient to show that there exists a positive constant $\mu$ such that for all $\Phi(x) \in \tilde{W}(-1,1)$

$$
-\int_{-1}^{1} \int_{-1}^{1} \ln |x-y| \Phi^{\prime}(x) \Phi^{\prime}(y) d x d y \geq \mu \int_{-1}^{1} \Phi^{2}(x) d x .
$$

The proof of (5) is based on the expansion of $\Phi$ into the series of weighted Chebyshev's polynomials

$$
\Phi(x)=\sum_{n=0}^{\infty} \phi_{n} U_{n}(x) w(x), \quad w(x)=\sqrt{1-x^{2}},
$$

with $U_{n}(\cos \theta)=\sin (n+1) \theta / \sin \theta$. Using formula (3) and the following properties of Chebyshev's polynomials [12]

$$
-\int_{-1}^{1} \frac{\left(U_{n}(y) w(y)\right)^{\prime}}{y-x} d y=\pi(n+1) U_{n}(x), \quad \int_{-1}^{1} U_{n}(x) U_{m}(x) w(x) d x=\frac{\pi}{2} \delta_{m n},
$$

one can then transform the left-hand side of (5) to

$$
-\int_{-1}^{1} \int_{-1}^{1} \ln |x-y| \Phi^{\prime}(x) \Phi^{\prime}(y) d x d y=\sum_{m, n=0}^{\infty} A_{m n} \phi_{m} \phi_{n}=(A \phi, \phi),
$$

where $A$ is the diagonal matrix of infinite dimension with the elements

$$
A_{m n}=\frac{\pi^{2}}{2}(m+1) \delta_{m n}
$$

The obvious consequence of $(8)$ is that the quadratic form $(A \phi, \phi)$ is positive definite. On the other side,

$$
\int_{-1}^{1} \Phi^{2}(x) d x=\sum_{m, n=0}^{\infty} B_{m n} \phi_{m} \phi_{n}=(B \phi, \phi),
$$

with

$$
\begin{aligned}
B_{m n} & =\int_{-1}^{1} U_{m}(x) U_{n}(x)\left(1-x^{2}\right) d x= \\
& = \begin{cases}0 & \text { if } m+n \text { is odd, } \\
\frac{4(m+1)(n+1)}{(m+n+3)(m+n+1)(m-n+1)(n-m+1)} & \text { otherwise. }\end{cases}
\end{aligned}
$$

Mention that the quadratic form $(B \phi, \phi)$ is also symmetric and positive definite. 

quotient

Let us consider the variational problem of finding the smallest value of Rayleigh's

$$
\min _{\phi \neq 0} \frac{(A \phi, \phi)}{(B \phi, \phi)}=\mu \text {. }
$$

Obviously, $\mu$ is the smallest eigenvalue of the generalized eigenvalue problem

$$
(A-\mu B) \phi=0,
$$

or, equivalently, $\lambda=1 / \mu$ is the largest eigenvalue of the matrix $B A^{-1}$. This problem is solved with the help of Mathematica, and the calculation shows that $\lambda$ converges quickly to the numerical value $\lambda=0.274933$ (already with $15 \times 15$-matrix). So, the inequality (5) is valid for $\mu=3.63725$, and the equality is achieved if $\phi=\phi^{(1)}, \phi^{(1)}$ being the corresponding eigenvector. The graph of $\Phi(x)=\sum \phi_{n}^{(1)} U_{n}(x) w(x)$ is plotted in Fig. 1.

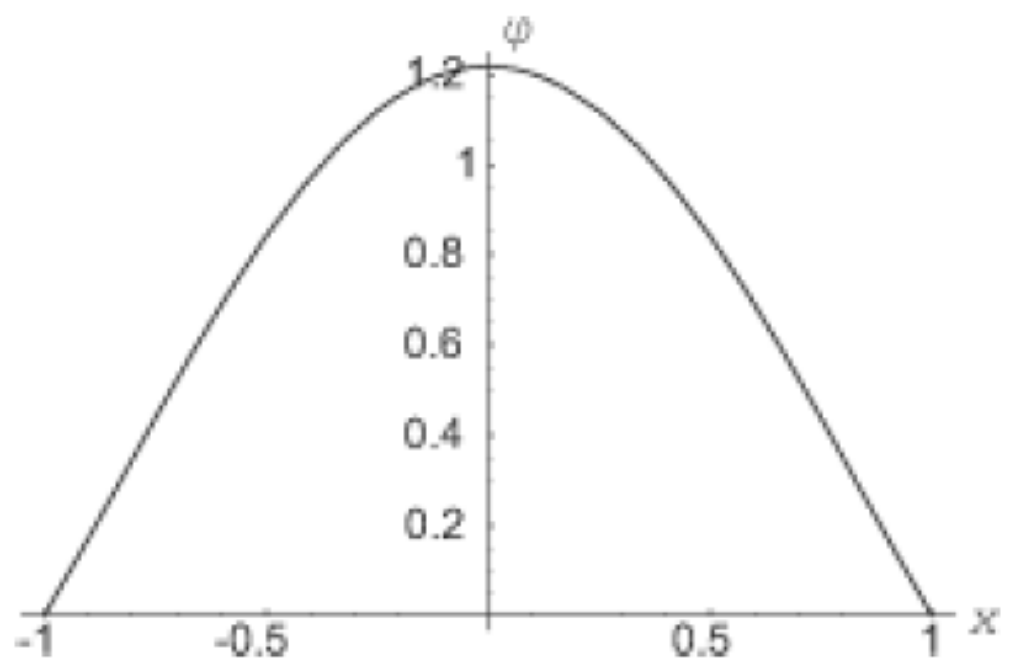

Fig. 1. The graph of function $\Phi(x)$ for which (5) becomes an equality

Note that the analogous inequality for $\Phi(x) \in W^{1,2}(\mathbb{R})$ does not exists. Indeed, for $\Phi(x) \in W^{1,2}(\mathbb{R})$ we have

$$
-\int_{-\infty}^{\infty} \int_{-\infty}^{\infty} \ln |x-y| \Phi^{\prime}(x) \Phi^{\prime}(y) d x d y=\int_{-\infty}^{\infty} H\left(\Phi^{\prime}\right)(x) \Phi(x) d x
$$

where $H(f)$ denotes the Hilbert transform of the function $f(x)$. Using the property of the Hilbert and Fourier transforms we can write this in the form

$$
-\int_{-\infty}^{\infty} \int_{-\infty}^{\infty} \ln |x-y| \Phi^{\prime}(x) \Phi^{\prime}(y) d x d y=\int_{-\infty}^{\infty}|t| \tilde{\Phi}^{2}(t) d t
$$


where $\tilde{f}(t)$ denotes the Fourier transform of $f(x)$. Thus, the quadratic form (9) is positive definite. On the other side

$$
\int_{-\infty}^{\infty} \Phi^{2}(x) d x=\int_{-\infty}^{\infty} \tilde{\Phi}^{2}(t) d t
$$

due to the Plancherel identity. Thus, the inequality

$$
\int_{-\infty}^{\infty}|t| \tilde{\Phi}^{2}(t) d t \geq \mu \int_{-\infty}^{\infty} \tilde{\Phi}^{2}(t) d t
$$

would be violated by the functions $\tilde{\Phi}(t)$ which are positive inside the small interval $(-\varepsilon, \varepsilon)$ and vanish outside it.

\section{REFERENCES}

[1] S. Nemat-Nasser and M. Hori. Toughening by partial or full bridging of cracks in ceramics and fiber reinforced composites. Mechanics of Materials, 6 (1987), 245-269.

[2] M. Hori and S. Nemat-Nasser. Asymptotic solution to a class of strongly singular integral equations. Journal of Applied Mathematics, 50 (1990), 716-725.

[3] J. R. Willis and S. Nemat-Nasser. Singular perturbation solution of a class of singular integral equations. Quarterly of Applied Mathematics, 48 (1990), 741-753.

[4] A. K. Gautesen. On the solution to a class of strongly singular linear integral equations. Quarterly of Applied Mathematics, 50 (1992), 129-140.

[5] A. B. Movchan and J. R. Willis. Asymptotic analysis of the reinforcement of a brittle crack by bridging fibres. Quarterly Journal of Mechanics and Applied Mathematics, 46 (1993), 331-350.

[6] K. C. Le and H. Stumpf. On the cohesive crack model. ZAMM, 75 (1995), 231-232.

[7] J. D. Eshelby. Edge dislocations in anisotropic materials. Phil. Mag., 40 (1949), 903-912.

[8] G. Leibfried. Verteilung von Versetzungen im statischen Gleichgewicht. Z. Phys., 130 (1951), 214-226.

[9] J. P. Hirth and J. Lothe. Theory of Dislocations. John Wiley, New York (1982).

[10] J. F. Toland. The Peierls-Nabarro and Benjamin-Ono equations. J. Funct. Anal., 145 (1997), $136-150$.

[11] V. Karlin, V. G. Mazya, A. B. Movchan, J. R. Willis, and R. Bullough. Numerical solution of nonlinear hypersingular integral equations of the Peierls type in dislocation theory. Siam J. Appl. Math., 60 (2000), 664-678.

[12] A. Erdelyi, W. Magnus, F. Oberhettinger, and F. G. Tricomi. Higher transcendental functions, volume II. McGraw-Hill, New York (1953).

Received July 27, 2009

\section{BẤT ĐẲNG THỨC CỦA MộT PHIẾM HÀM BẬC HAI}

Một bất đẳng thức được chứng minh cho một phiếm hàm bậc hai với nhân lôgarít. Các hằng số tốt nhất của bất đẳng thức này và hàm tương ứng với đẳng thức xảy ra được tìm thấy một cách chính xác. 